Article

\title{
Physical Characterization of Cementitious Materials on Casting and Placing Process
}

\section{Hong Jae Yim ${ }^{1}$ and Jae Hong Kim ${ }^{2}$ *}

1 Department of Civil and Environmental Engineering, Korea Advanced Institute of Science and Technology, Daejeon 305-701, Korea; E-Mail: yimhongjae@gmail.com

2 School of Urban and Environmental Engineering, Ulsan National Institute of Science and Technology, Ulsan 689-798, Korea

* Author to whom correspondence should be addressed; E-Mail: jaekim@ unist.ac.kr; Tel.: +82-52-217-2817; Fax: +82-52-217-2819.

Received: 25 February 2014; in revised form: 7 April 2014 / Accepted: 10 April 2014 / Published: 15 April 2014

\begin{abstract}
Coagulation of cement particles is an inevitable phenomenon of fresh cement-based materials undergoing solidification. Coagulation can be classified into two types, reversible flocculation and irreversible coagulation, wherein microstructural change affects the rheological properties, including shear thinning and thixotropy, and the hydration process. This paper attempts to measure the mechanical property and the coagulation of cement particles according to the mix proportions of cement paste. Experimental setups were proposed for two different types of coagulations using a laser backscattering instrument. Volume fraction and size distribution of coagulated particles were obtained, and their variations were discussed. From the obtained results the microstructural buildup of freshly mixed cement pastes can be divided into three categories: permanent coagulation and strong and weak flocculation.
\end{abstract}

Keywords: cement paste; particle size; coagulation; flocculation; laser backscattering

\section{Introduction}

Cementitious materials, including portland cement, pozzolans and granulated slag, are extensively consumed for building infrastructures these days. The cementitious material provides binding properties to compose bulk engineering materials, of which the majority of the volume is occupied by 
inert fillers, such as natural aggregates. Its binding property is manifested by the hydration of cementitious material, and its aqueous suspension temporarily exists as a precursory state. The time of suspension is called the dormant period because the chemical reaction to produce binding compounds momentarily pauses before its activation. In the case of portland cement, the time for which the powder-type cementitious material can be suspended is more than $1 \mathrm{~h}$, and the solidification of suspension is then followed with the passage of time. For engineering practices, the period of suspension is critical for the casting and placing process of the cement-based materials, which is the only time which allows us to cast products. Therefore understanding of the suspension of cementitious materials enhances the casting and placing performance of cementitious composites and, consequently, secures the quality of infrastructure materials.

One of the most principal phenomena in the suspension of cementitious materials is coagulation, a process of dispersed particles growing in the form of clusters [1]. Pulverized cement in water involves combination and adhesion of cement particles due to potential energy of van der Waals attraction [2], which results in coagulation, solid percolation in the suspension, and, finally, solidification with cement hydration. Notably, such a change of the fresh microstructure affects cement hydration and the rheological properties, including shear thinning and thixotropy [3].

The coagulation can be classified into two types, reversible or irreversible phenomenon. The reversible coagulation is generally referred to as flocculation, and flocculated particles can be separated by an applied external power larger than their flocculation strength. When fresh cement-based material is under high shear flow and high pressure, such as in the case of pumping and vibrated consolidation, the flocculated cement particles are broken in practice. In contrast, irreversible coagulation leaves permanent coagulates that cannot be broken by any physical agitation. The permanent coagulation is due to quick setting of cementitious materials, which is related to the monosulfate and ettringite formation with the hydration of aluminate $\left(3 \mathrm{CaO} \cdot \mathrm{Al}_{2} \mathrm{O}_{3}\right)$. Even in the dormant period of lime-silica compounds $\left(3 \mathrm{CaO} \cdot \mathrm{SiO}_{2}\right.$ or $\left.2 \mathrm{CaO} \cdot \mathrm{SiO}_{2}\right)$, holding a large majority in cementitious materials, the aluminate hydration activates on the coagulated particles and tightly ties them. Its amount of reactive products is small but critical to control the early-age stiffening of suspension, which decreases its consistency.

The kinematics of cement particles in aqueous suspension can be monitored using a laser backscattering instrument. Cement particles and clusters reflect the incident laser and the delay intensity of the backscattered laser indicates the chord length of the particles in suspension. Its in situ applications in a reactor have been widely reported in the field of chemical engineering [4-9]. For a neat cement paste, it was found that the flocculation is affected by the alkalis/alumina content, and nanoclay is one of the most influential admixtures with respect to the flocculation rate [10,11]. Furthermore, the effect of the flocculant on the fiber-cement suspension was observed during the manufacturing process [12-14].

Previous studies on cement-based materials show the possibility of quantitative measurement of cement coagulation, where only the mean size $(\mu)$ of cement flocs was monitored to reveal the effects of admixtures and chemical contents. The amount of coagulation and their size distribution have not been quantitatively monitored, even though researchers presume that coagulation increases with the passage of time. This paper attempts to measure the coagulation of portland cement particles and the effect of mix proportions is also investigated. Two different types of coagulation, permanent 
coagulation and flocculation, are separately measured with two different setups proposed in the study. From the obtained results, their volume fraction $(\phi)$ and size distribution are obtained and the microstructural buildup of freshly mixed cement pastes is revealed.

\section{Experiment}

\subsection{Sample Preparation}

A total of five cement paste mixes were prepared with ordinary Type I portland cement. The specific gravity and specific surface determined by the Blaine method are 3.14 and $3320 \mathrm{~cm}^{2} / \mathrm{g}$, respectively. Table 1 shows the oxide composition of the used powders.

Table 1. Oxide composition of the cement.

\begin{tabular}{cccccccccc}
\hline Oxide & $\mathbf{C a O}$ & $\mathbf{S i O}_{\mathbf{2}}$ & $\mathbf{A l}_{\mathbf{2}} \mathbf{O}_{3}$ & $\mathbf{M g O}$ & $\mathbf{S O}_{3}$ & $\mathbf{F e}_{2} \mathbf{O}_{3}$ & $\mathbf{K}_{\mathbf{2}} \mathbf{O}$ & $\mathbf{T i O}_{2}$ & $\mathbf{N a}_{2} \mathbf{O}$ \\
\hline Percent $(\%)$ & 63.6 & 19.2 & 4.8 & 3.8 & 3.7 & 3.0 & 1.1 & 0.3 & 0.2 \\
\hline
\end{tabular}

The mix proportion of each sample to obtain a $30 \mathrm{~mL}$ volume is described in Table 2, where two comparison sets are found according to the water-to-cement ratio $(w / \mathrm{cm})$ and the dosage of high-range water-reducing admixture (HRWRA). The $w / \mathrm{cm}$ by mass was $40 \%, 30 \%$, and $60 \%$ for mixes $\mathrm{C} 1, \mathrm{C} 4$, and $\mathrm{C} 5$, respectively. The effect of HRWRA was also investigated in mixtures having a water-to-powder ratio of $40 \%$ (mixes $\mathrm{C} 1, \mathrm{C} 2$, and $\mathrm{C} 3$ ). The used HRWRA was a polycarboxylate-based admixture with a solid content of $30 \%$ by mass (ADVA 128, W. R. Grace \& Co., Columbia, MD, USA). The HRWRA dosage was $0.2 \%$ and $0.35 \%$ of cement mass, which are, respectively, labeled $\mathrm{C} 2$ and $\mathrm{C} 3$, while no HRWRA is mixed for $\mathrm{C} 1$. All samples were produced according to the following mixing procedure: (1) hand-mixing for $2 \mathrm{~min}$; (2) scraping the mixing beaker (100 $\mathrm{mL}$ volume) for $1 \mathrm{~min}$; and (3) hand-mixing again for $2 \mathrm{~min}$. The room temperature $\left(22^{\circ} \mathrm{C}\right)$ and humidity $(50 \%)$ were maintained during the mixing and following experiment.

Table 2. Mix proportions of prepared samples.

\begin{tabular}{ccccc}
\hline Label & $\boldsymbol{w} / \mathbf{c m}(\boldsymbol{\%})$ & Water $(\mathbf{g})$ & Cement $(\mathbf{g})$ & HRWRA $(\mathbf{g})$ \\
\hline C1 & 40 & 17 & 42 & - \\
C2 & 40 & 17 & 42 & 0.084 \\
C3 & 40 & 17 & 42 & 0.146 \\
C4 & 30 & 15 & 49 & - \\
C5 & 60 & 20 & 33 & - \\
\hline
\end{tabular}

\subsection{Flow Curves Determination}

The flow curves of the samples were measured to determine their rheological properties. A commercialized rheometer (HAAKE MARS III, Thermo Fisher Scientific Inc., Waltham, MA, USA) was used. The measuring geometry of the rheometer was parallel plates with a diameter of $35 \mathrm{~mm}$ and the gap between the plates was set to $1 \mathrm{~mm}$. The rheological measurement was obtained immediately after the mixing was finished. The protocol for the measurement followed ASTM C1749 [15]. After 1 min pre-shearing at $100 \mathrm{~s}^{-1}$, the step of strain rate was decreased as $80 \mathrm{~s}^{-1}, 60 \mathrm{~s}^{-1}, 40 \mathrm{~s}^{-1}, 20 \mathrm{~s}^{-1}$, 
$10 \mathrm{~s}^{-1}, 5 \mathrm{~s}^{-1}, 1 \mathrm{~s}^{-1}, 0.5 \mathrm{~s}^{-1}$, and $0.1 \mathrm{~s}^{-1}$, sequentially. The shear stress was measured for $20 \mathrm{~s}$ at each step, and the final shear stress was obtained when the value became stable. The measured flow curves were described in the next section. Each $30 \mathrm{~mL}$ sample was encapsulated to prevent water evaporation and maintained in a static condition. A small amount of the encapsulated sample was taken and experimented on in series. Different experimental procedures were proposed depending on the phenomenon to be characterized: (1) flocculation and (2) permanent coagulation. Details are described in the next section.

\subsection{Laser Backscattering Instrument}

Monitoring dimensional information of particles in a suspension was accomplished with a laser backscattering instrument (ORM, optical-back reflectance measurement, Sequip GmbH, Düsseldorf, Germany). Through the ORM probe, a laser beam with an intensity of $3 \mathrm{~mW}$ is emitted. The laser beam is dynamically focused on the front head, which is covered with Sapphire glass, having a diameter of $17.8 \mathrm{~mm}$. The dynamic focus follows a three-dimensional elliptical orbit, where it makes an $8.5 \mathrm{~mm}$-diameter circle horizontally and also moves in a normal direction, dynamically set at $40 \mu \mathrm{m}$ to $125 \mu \mathrm{m}$ above the window. The laser optic system obtains the scattered time of the laser signals and then determines the length of particles and their population. As a result, the number and length distribution of the particles are measured within a range of $0.5 \mu \mathrm{m}$ to $1200 \mu \mathrm{m}$. The resolution is $0.5 \mu \mathrm{m}$. The unweighted size distribution can also be converted into the area or volume fraction [8].

\subsection{Experiment for Permanent Coagulation}

To investigate permanent coagulates in cement pastes it is necessary to first eliminate breakable (reversible) particles in the samples. Each sample was diluted with isopropyl alcohol as follows: $4 \mathrm{~mL}$-paste in $400 \mathrm{~mL}$-alcohol (1\% volume fraction). The use of alcohol prevents cement hydration and particle interaction. Alcohol is also expected to help flocs fragment quickly. The diluted sample was stirred for $5 \mathrm{~min}$ at 900 RPM to break the clusters. Here, it is assumed that the flocculation strength is less than the shear energy applied at 900 RPM. Successive stirring was carried out at 600 RPM, where the particle size distribution was measured after the flow in a beaker became stable. The time to obtain a stable flow and, thus, consistent measurement was less than $10 \mathrm{~min}$. In the subsequent $10 \mathrm{~min}$ period, the size distribution of permanent coagulates was measured with an increment of $1 \mathrm{~min}$ and the average value is reported.

Figure 1 shows the conventional top-down setup and the diluted sample is placed in a $1 \mathrm{~L}$ beaker (110 $\mathrm{mm}$ diameter by $150 \mathrm{~mm}$ height). An impeller, tilted at $20^{\circ}$ to avoid turbulence of the suspension, is located $10 \mathrm{~mm}$ above the bottom of the beaker. A half-moon axial impeller is used in the study, which is composed of two collapsible blades made with PTFE and its size is $65 \mathrm{~mm} \times 18 \mathrm{~mm} \times 3 \mathrm{~mm}$. According to the agitating procedure described above, the diluted sample to be tested is agitated with an overhead stirrer (WiseStir, HT-120DX, Wertheim, Germany). 
Figure 1. Top-down setup for permanent flocculates.

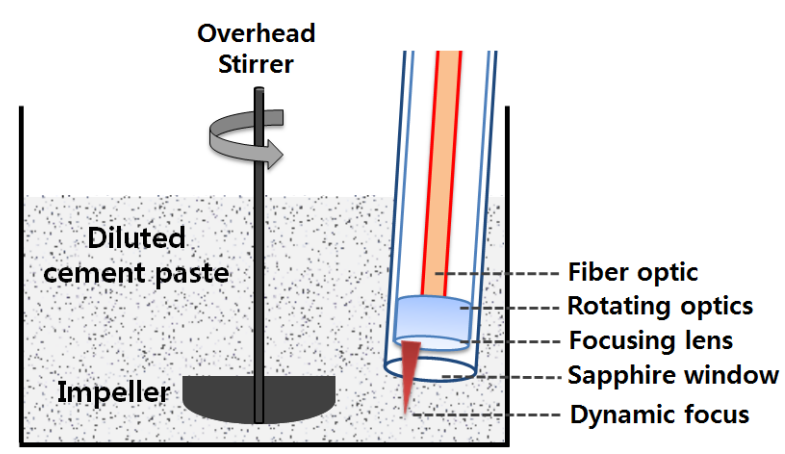

\subsection{Experiment for Flocculation}

Flocculation strength is various in cement paste. At a certain shear rate, some clusters, having a small flocculation strength, are broken while others, having a large flocculation strength, are not. At higher agitating speeds, more clusters are broken into small particles. On the other hand, the particles that partially deflocculated are too large (heavy) to float up at low-speed agitation. Increase of the agitating speed causes both deflocculation and floating large particles, which results in decreased particle size and quantity (count) of particles at the bottom of suspension. In order to capture these phenomena, a bottom-up setup for water dilution was used. A $1.5 \mathrm{~mL}$ sample was diluted in $150 \mathrm{~mL}$ of water ( $1 \%$ volume fraction) for measurement. The deflocculation ratio was measured while the agitating speed was gradually increased from 500 to 900 RPM in increments of 100 RPM. At each RPM, the number and size distribution of particles were measured during $10 \mathrm{~s}$ to stabilize the bottom-up setup for measurement of flocculation.

Figure 2 schematically shows the experimental setup where the ORM probe is placed bottom-up to a diluted sample. The diluted sample is placed in a fiber-pipe container having a diameter of $53 \mathrm{~mm}$. The ORM probe is located at the bottom of the container and its axis is aside from the central axis of the container. The 150-mL diluted sample fills the container and the sample height reaches $69 \mathrm{~mm}$. After the sample is placed, an impeller is put at the center of the bottom. The measurement procedure is then followed with the same impeller and agitator.

Figure 2. Bottom-up setup for flocculates.

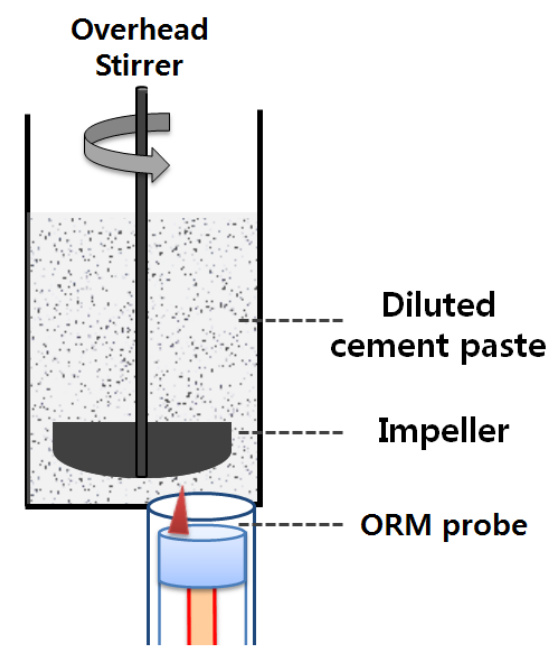




\section{Results and Discussion}

\subsection{Flow Curves of the Samples}

Figure 3 shows the data points measured at each step, according to the protocol and their trend lines are also plotted. Each flow curve is the average of three measurements with their replicated samples. The yield stress of all samples is not significant and their flow curves follow a power law function. The use of HRWRA or higher water-to-cement ratio decreases the viscosity, as expected.

Figure 3. Flow curves of the samples according to the (a) different HRWRA dosage and (b) different $w / \mathrm{cm}$.

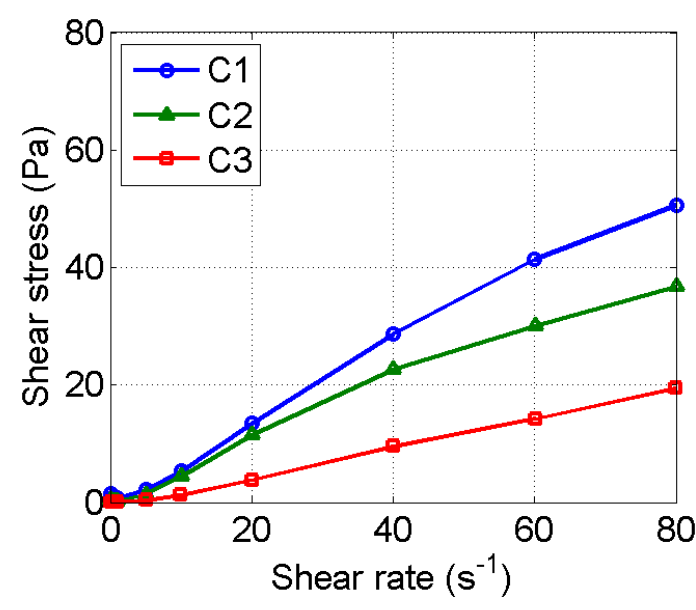

(a)

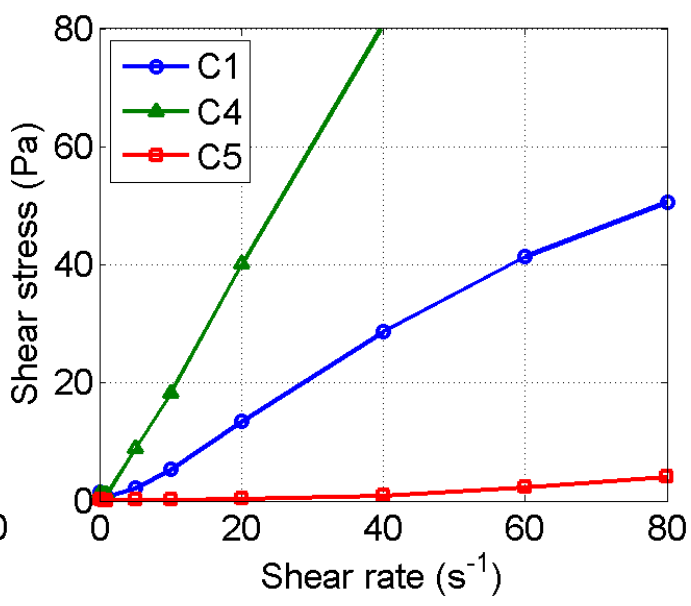

(b)

\subsection{Raw Powder}

Prior to the measurement of cement paste, the size distributions of raw cement powder were measured. Alcoholic dilution and the top-down conventional setup for permanent coagulation were used to measure the diameters $(d)$ of each grain. Based on the measurement data, the probability distribution (number distribution) of grain diameter is achieved. The volume fraction of the powder can be calculated by multiplying the probability distribution by the cube of diameter $d^{3}$. While the probability distribution represents the counts at intervals of their diameter, the volume fraction describes the volume fraction at each interval. The distribution of solid volume fraction explains the packing and rheology of the pastes. Figure 4 shows the measured probability distribution and its volume fraction. The volume fraction of the cement powder hits the local minimum at about $34 \mu \mathrm{m}$ and it rises. In contrast, the particle counts of which diameter is larger than $10 \mu \mathrm{m}$ are negligible in the probability distribution. Even a very small amount of large particles highly influences the volume fraction and packing. In the current data, the particles larger than $10 \mu \mathrm{m}$ are due to cement agglomeration and it is natural in practical storage of cement powder.

In this research, the grain diameter of raw cement powder is assumed to follow a lognormal distribution, which is a common type of probability distribution for raw cement powder size. In other words:

$$
d \sim \operatorname{LN}\left(\mu_{\mathrm{c}}, \sigma_{\mathrm{c}}\right)
$$


where $d$ is the grain diameter and $\operatorname{LN}\left(\mu_{\mathrm{c}}, \sigma_{\mathrm{c}}\right)$ denotes the lognormal distribution with mean $\mu_{\mathrm{c}}$ and standard deviation $\sigma_{\mathrm{c}}$. The powder grains smaller than $10 \mu \mathrm{m}$ are less sensitive to the large particles agglomerated. Therefore, their mean and standard deviation within the range were evaluated as reported by Table 3 (raw grains).

Figure 4. Size distribution of cement powder: (a) the measured number distribution and (b) the converted volume distribution.

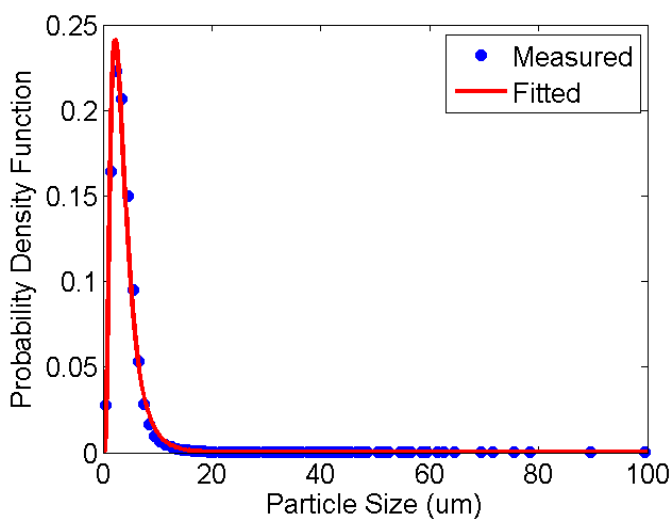

(a)

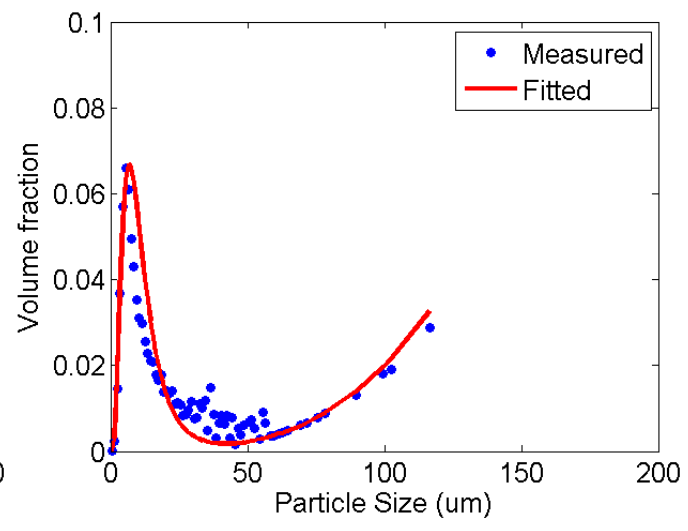

(b)

Table 3. Size distribution of the cement powder.

\begin{tabular}{ccc}
\hline Diameter distribution, $\boldsymbol{\mu m}$ & Raw grains & Agglomerates \\
\hline mean & 3.84 & 7.74 \\
standard deviation & 2.33 & 1.59 \\
\hline
\end{tabular}

The large-particles tail in the volume fraction is apt for investigating agglomeration. The assumption that the agglomeration does not change the total volume of solid particles provides a complementary volumetric relation on the agglomeration. This corresponds to the fractal dimension of 3, where the floc porosity is constant during coagulation [16]. In such a situation, the decrease in the volume of raw cement powders $\left(1-\phi_{a}\right)$ is the same as the increase in the total volume of their agglomerates $\left(1-\phi_{\mathrm{a}}\right)$. Another lognormal distribution for large agglomerates is introduced to Equation (1) for describing the probability density function of grain diameter:

$$
d \sim \phi_{\mathrm{a}} \cdot \mathrm{LN}\left(\mu_{\mathrm{a}}, \sigma_{\mathrm{a}}\right)+\left(1-\phi_{\mathrm{a}}\right) \cdot \mathrm{LN}\left(\mu_{\mathrm{c}}, \sigma_{\mathrm{c}}\right)
$$

where $d$ is the grain diameter, $\phi_{\mathrm{a}}$ is the total volume fraction, and $\mu_{\mathrm{a}}$ and $\sigma_{\mathrm{a}}$ are the mean and standard deviation of the agglomerates diameter. The probability distribution in Equation (2) is a mixture distribution which is the weighted combination of two probability distributions. Equation (2) multiplied by the cube of diameter $d^{3}$ is fitted to the volume fraction using nlinfit, a MATLAB ${ }^{\circledR}$ function for nonlinear regression analysis so that the least square error between the measured data and the fitted probability distribution was minimized. The three unknown parameters of the agglomerates were consequently obtained. Its total volume fraction $\left(\phi_{\mathrm{p}}\right)$ is 0.012 and its mean $\left(\mu_{\mathrm{a}}\right)$ and standard deviation $\left(\sigma_{\mathrm{a}}\right)$ are reported in Table 3 (agglomerates). In addition, in Figure 4, the fitted curve is shown by red lines. In the next section, it will be shown that the distribution of permanent coagulation and flocculation has a greater portion of the tail of large particles. 


\subsection{Permanent Coagulation}

Coagulation accelerates when the cement paste samples in air are at rest. Sometimes, it is called agglomeration. The dimensional information of permanent coagulates was measured with a top-down conventional setup with alcoholic dilution. Fitting the volume fraction provides quantitative information on the permanent coagulation. Figure 5 shows the change in the volume fraction of mix $\mathrm{C} 1$, where the size of permanent coagulates is increasing with the passage of time at rest. The volume distribution at $4 \mathrm{~h}$ is very similar with the 2-h-measurement, except that the small size peak (at around $10 \mu \mathrm{m})$ is slightly decreased and the large size tail is more tip-tilted.

Figure 5. Volume distributions of mix $\mathrm{C} 1$ at (a) $0 \mathrm{~h}$ and (b) $2 \mathrm{~h}$ after mixing.

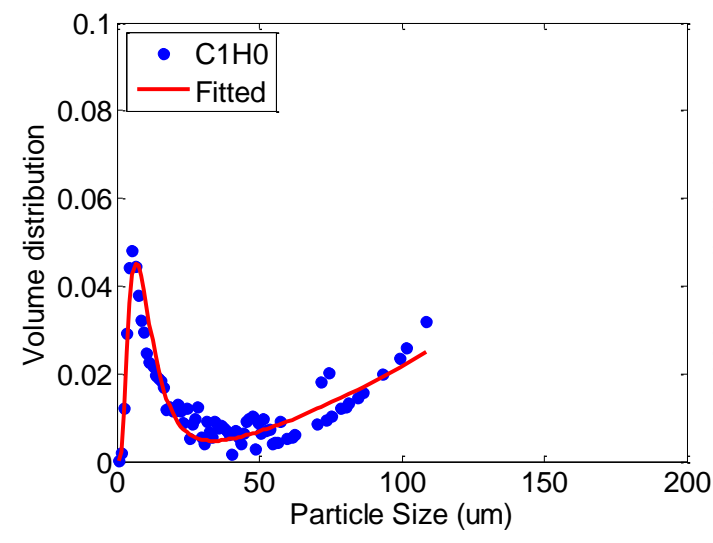

(a)

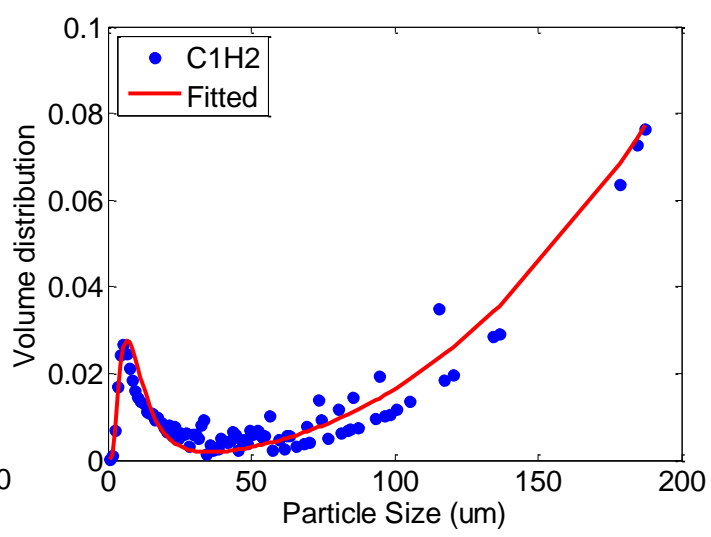

(b)

The assumption of the complementary volumetric relation is also applied on the coagulation. Another lognormal distribution for permanent coagulates is here introduced:

$$
d_{\mathrm{p}} \sim \phi_{p} \cdot \mathrm{LN}\left(\mu_{\mathrm{p}}, \sigma_{p}\right)+\left(1-\phi_{p}\right) \cdot \mathrm{LN}\left(\mu_{\mathrm{c}}, \sigma_{\mathrm{c}}\right)
$$

where $d_{\mathrm{p}}$ is the grain diameter after coagulation, $\phi_{\mathrm{p}}$ the total volume fraction, and $\mu_{\mathrm{p}}$ and $\sigma_{\mathrm{p}}$ are the mean and standard deviation of the permanent coagulates diameter. Then, it is fitted again with nlinfit, a MATLAB ${ }^{\circledR}$ function. Table 4 reports the increase in the volume fraction of permanent coagulates. A threshold of $w / \mathrm{cm}$ exists at which a mix provides a consistent amount of permanent coagulation. In mixes $\mathrm{C} 1(w / \mathrm{cm} 40 \%)$ and $\mathrm{C} 4(w / \mathrm{cm} 30 \%)$ the respective amounts of permanent coagulation for $4 \mathrm{~h}$ were $18 \%$ and $14 \%$, respectively, but C5 $(w / \mathrm{cm} 60 \%)$ showed a value of about $5 \%$. Packing cement particles under such a low-threshold population provides a similar rate of permanent coagulation but decreasing the concentration of cement particles reduces the permanent coagulation.

Incorporating HRWRA reduces and decelerates permanent coagulation as found in mix C3. HRWRA is a polymer to increase the consistency of cement suspension when it is added in a very small amount (approximately $0.30 \%$ to $0.45 \%$ by weight of cement). It is composed of a hydrocarbon backbone having multiple polar groups and then easily attaches to cement particles. As a result, it causes deflocculation of cement particles and makes them hydrophilic. The deflocculation monitored in the superplasticized mixes, $\mathrm{C} 2$ and $\mathrm{C} 3$, will be discussed in the next section and the deflocculation also affects the permanent coagulation (retarding) as shown by high-dosage HRWRA mix C3. 
The mean sizes of permanent coagulates are shown in Figure 6, where the values are higher than that of raw cement powder $(3.84 \mu \mathrm{m})$ but sometimes smaller than that of agglomerates $(7.74 \mu \mathrm{m})$. The mixing process is thought to break the weak agglomeration and rebuild strong flocculation. However, their variation with time and the effect of mix proportion are not significant: within $4 \mathrm{~h}$, the maximum increase of mean size is only $7 \mu \mathrm{m}$ from $5 \mu \mathrm{m}$. This increase is not significant compared to the flocculation discussed in the next section.

Table 4. Volume fraction of permanent coagulation (\%).

\begin{tabular}{cccccc}
\hline Time (h) & Mix C1 & Mix C2 & Mix C3 & Mix C4 & Mix C5 \\
\hline 0 & 1.76 & 0.53 & 0.18 & 2.86 & 1.63 \\
2 & 4.28 & 2.8 & 0.35 & 4.3 & 3.51 \\
4 & 18.2 & 5.1 & 1.8 & 14.12 & 4.76 \\
\hline
\end{tabular}

Figure 6. Mean size of permanent coagulation (the dotted line is the mean of cement powder $\left.\left(\mu_{\mathrm{c}}\right), 3.84 \mu \mathrm{m}\right)$.

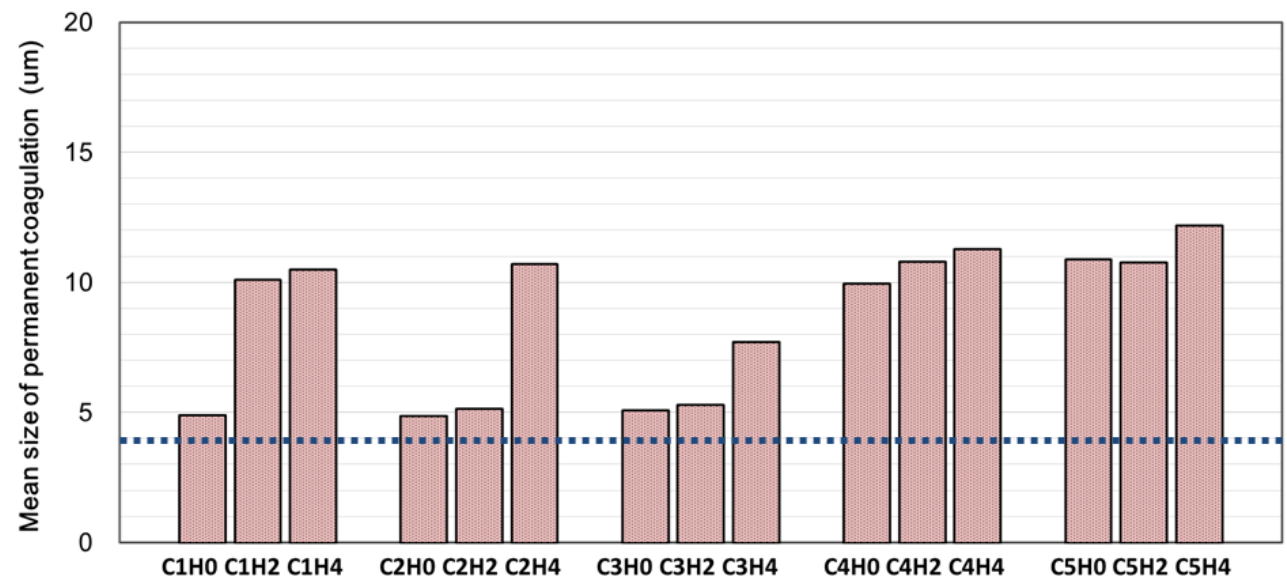

\subsection{Flocculation}

Dimensional information of flocculation is not easy to obtain because diluting a sample promptly causes deflocculation. Nevertheless, reversibility of flocculation allows us to use an indirect method: Measurement of deflocculation inversely indicates the rate of flocculation. The quantity and dimension of flocs are monitored during the process of deflocculation. For this purpose, a bottom-up setup for water dilution was used. Other factors affecting the rate of deflocculation, such as diluting concentration and its volume, are kept constant. The agitation speed is the only variable.

The quantity (count) of coagulated flocs was measured and its value decreases with increasing agitating speed from 500 to 900 RPM. Floating of deflocculated small particles results in a decrease of particle quantity at the bottom. Figure 7 shows the deflocculation ratio defined by the decreased ratio of the particle quantity at the 500 RPM reference measurement. For example, for the initial measurement (labeled by the suffix $\mathrm{H} 0$ ) of mix $\mathrm{C} 1,6 \%$ deflocculation was observed with the change of 500 RPM to 600 RPM. Further increase to 700 RPM, 800 RPM, and 900 RPM causes deflocculation up to $12 \%, 14 \%$, and $16 \%$, respectively. The passage of time at rest increases the amount of strong flocculation (900-RPM measurement), which is supported by higher deflocculation ratios for a 
few-hour-old samples. The samples were at rest for 2 and $4 \mathrm{~h}$, labeled by the suffixes $\mathrm{H} 2$ and $\mathrm{H} 4$, respectively. The only exception is mix C3 incorporating high-dosage HRWRA, where there is little change of the amount of flocculation over time and the amount is much higher than that of the others. A high dosage of HRWRA appears to restrain deflocculation during the mix process. In contrast, the deflocculation ratio of $\mathrm{C} 5(w / \mathrm{cm} 60 \%)$ is lower than that of $\mathrm{C} 4(w / \mathrm{cm} 30 \%)$ when measured before hydration (at $\mathrm{H} 0$ ), and a lower volume fraction of cement particles in the mix promotes deflocculation during the mixing process.

Figure 7. Deflocculation ratio of the cement pastes: (a) $\mathrm{C} 1$; (b) $\mathrm{C} 2$; (c) $\mathrm{C} 3$; (d) $\mathrm{C} 4$; and (e) $\mathrm{C} 5$, where the suffix $\mathrm{H} 0, \mathrm{H} 2$, and $\mathrm{H} 4$ implies the passage of time at rest.

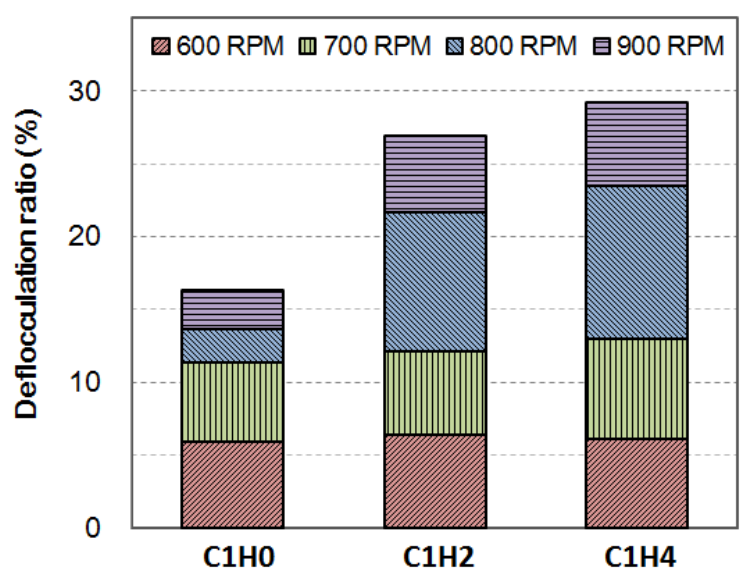

(a)

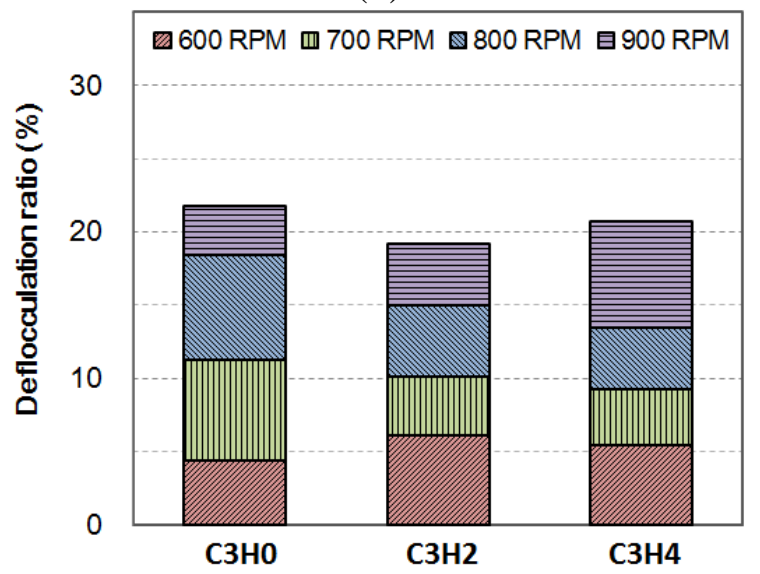

(c)

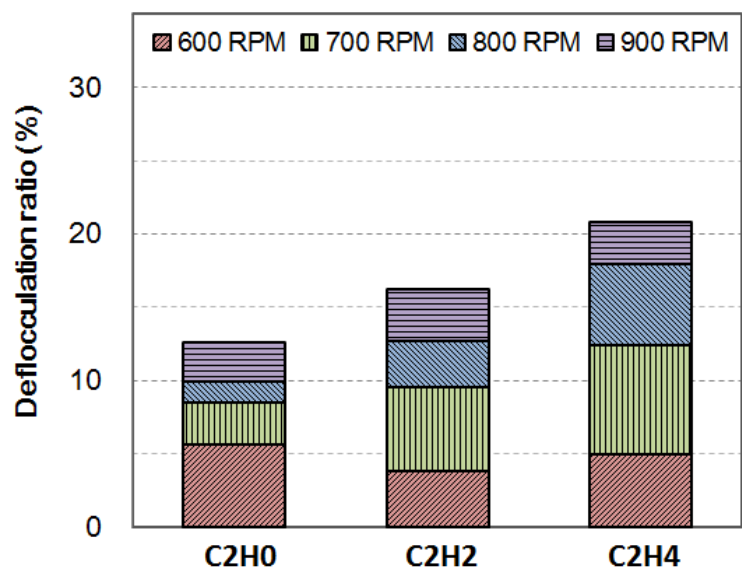

(b)

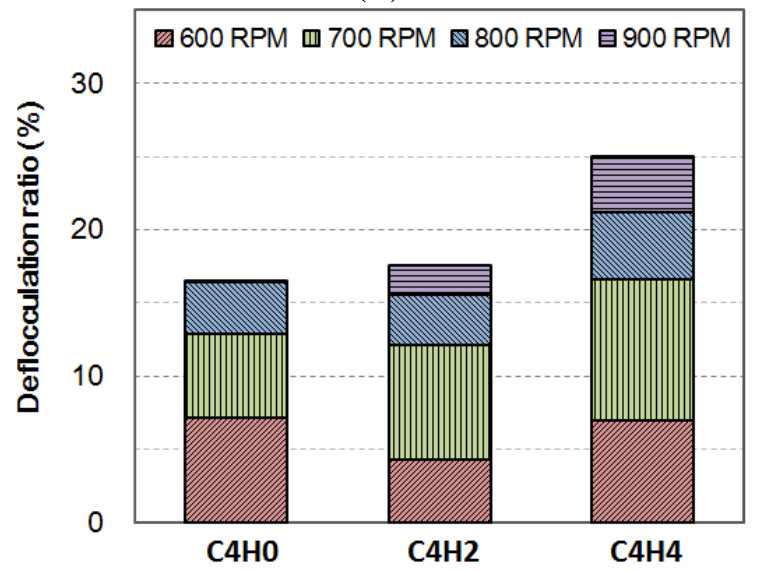

(d)

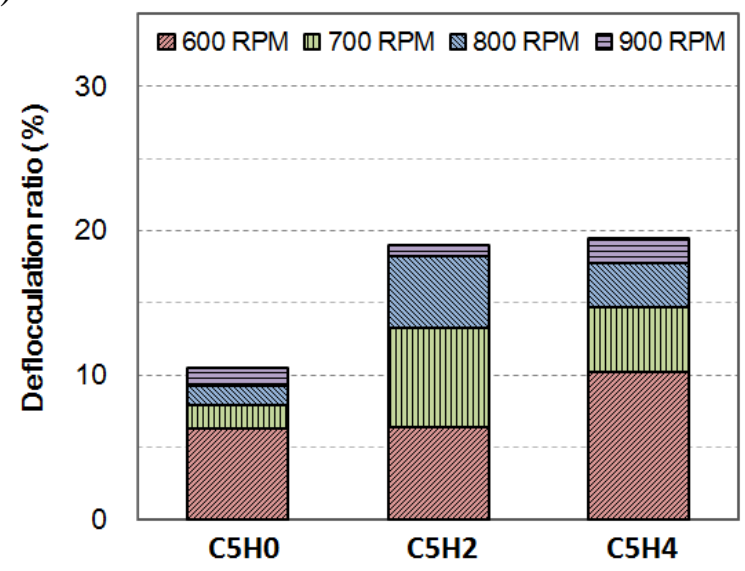

(e) 
Their volume fractions are also shown in Figure 8, where only the measurements at 500 RPM and 900 RPM are displayed for simplicity. At the 500 RPM measurement, small particles $(0-30 \mu \mathrm{m})$ observed in permanent flocculation (see Figure 5) are not found and large particles between 50 and $250 \mu \mathrm{m}$ dominate the volume fraction. The large particles generally increase with the passage of time. Mix C2 was an exceptional case, and shows that the use of HRWRA can reduce the degree of flocculation as the passage of time. On the other hand, applying high-speed agitation up to 900 RPM left-squeezes the volume fraction. In particular, the population of small particles distributed at approximately $10 \mu \mathrm{m}$ increases with 900 RPM. The loss of larger particles and increase of small particles confirms that deflocculation occurs in the measurement procedure. In addition, particles at all ages $(0,2$, and $4 \mathrm{~h}$ ) have similar size of less than $100 \mu \mathrm{m}$ under such high-speed agitation (900 RPM). The agitation did not provide sufficient power exceeding the flocculation strength at this level, indicating that the strong flocs asymptotically approach the state of permanent coagulates. The trend of particle size becomes similar with the volume fractions of permanent coagulate shown by Figure 5, where small particles between 1 and $50 \mu$ m reappear.

Figure 8. Volume distributions of mixes $\mathrm{C} 1$ to $\mathrm{C} 5$ (a) mix $\mathrm{C} 1$ at $500 \mathrm{RPM}$; (b) mix $\mathrm{C} 1$ at 900 RPM; (c) mix C2 at 500 RPM; (d) mix C2 at 900 RPM; (e) mix C3 at 500 RPM; (f) mix C3 at $900 \mathrm{RPM}$; (g) mix C4 at $500 \mathrm{RPM}$; (h) mix C4 at $900 \mathrm{RPM}$; (i) mix C5 at $500 \mathrm{RPM}$; (j) mix C5 at 900 RPM.

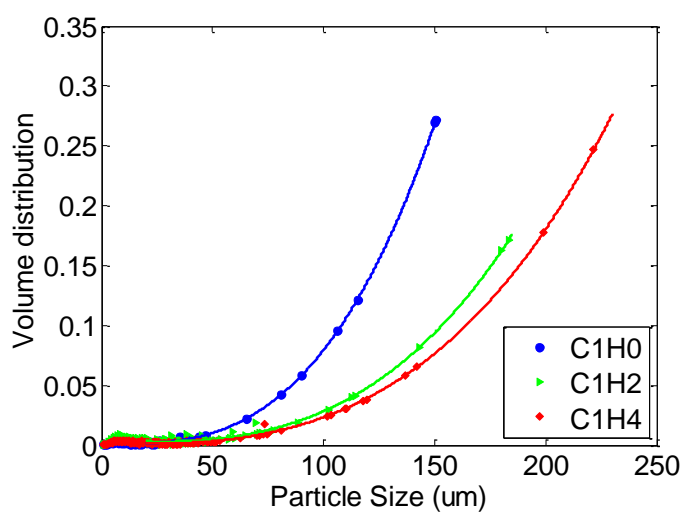

(a)

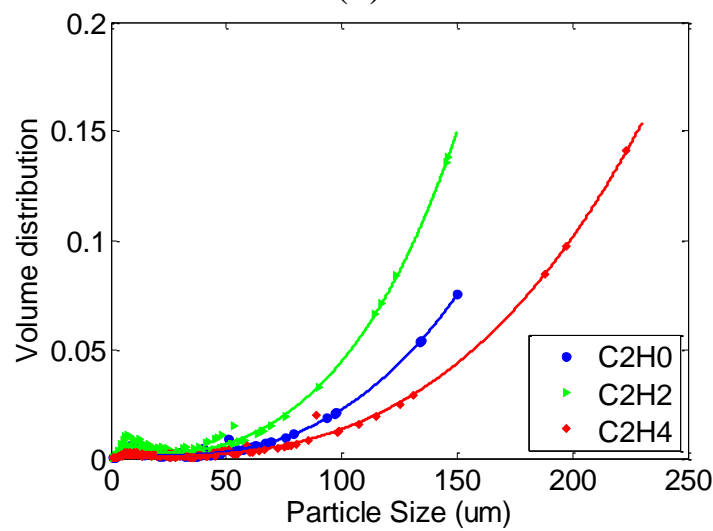

(c)

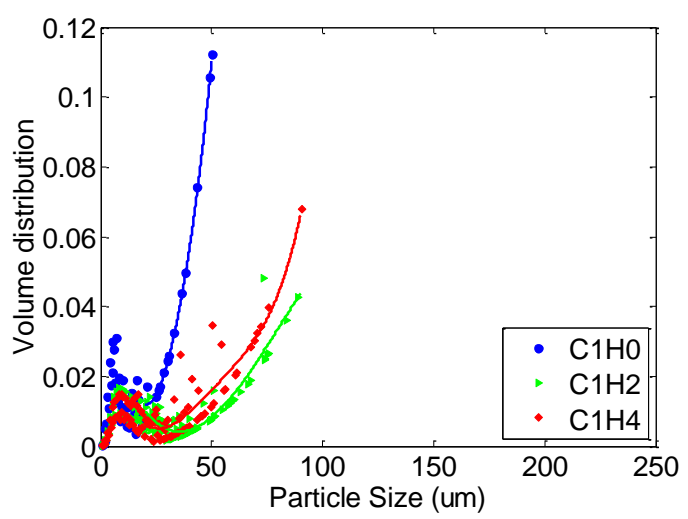

(b)

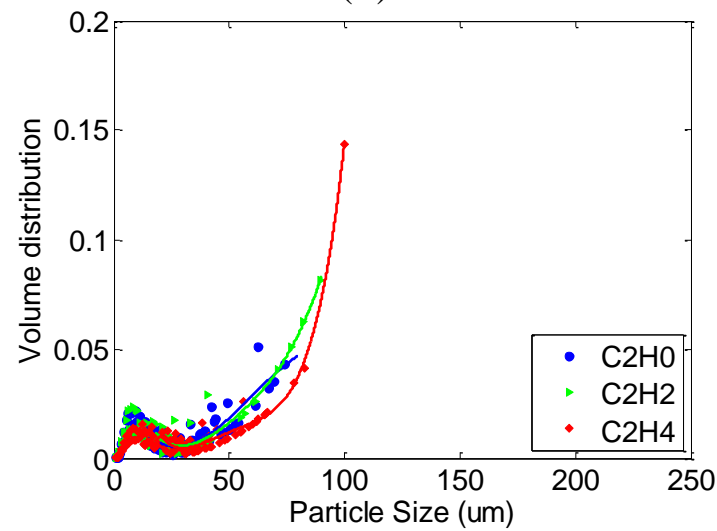

(d) 
Figure 8. Cont.

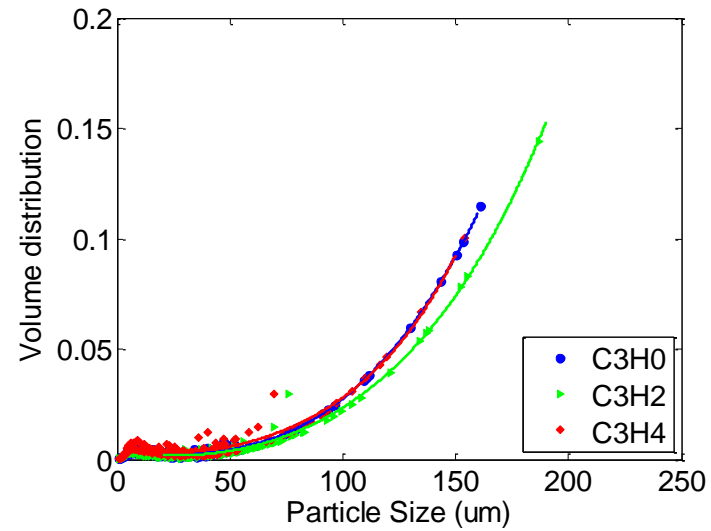

(e)

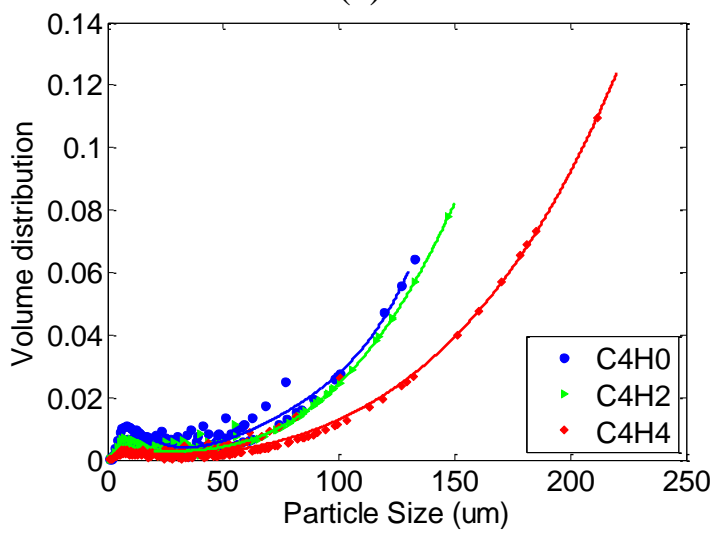

(g)

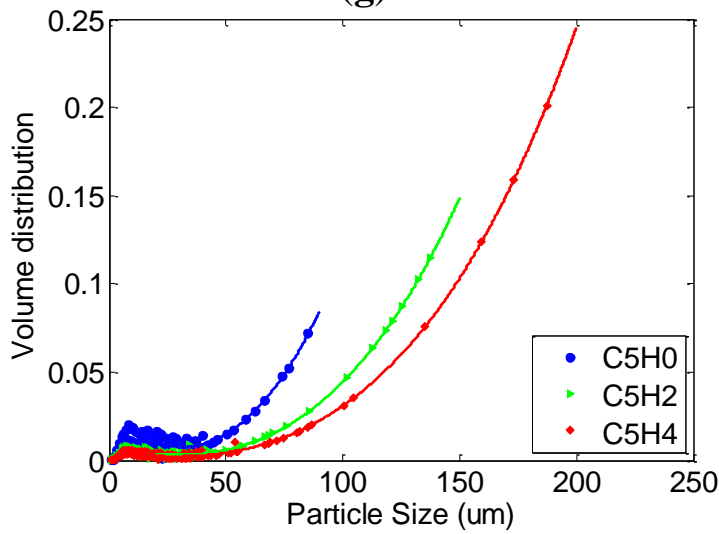

(i)

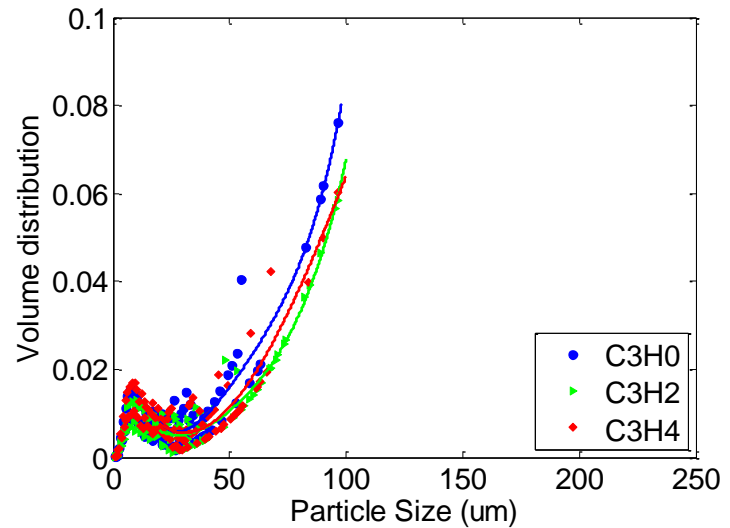

(f)

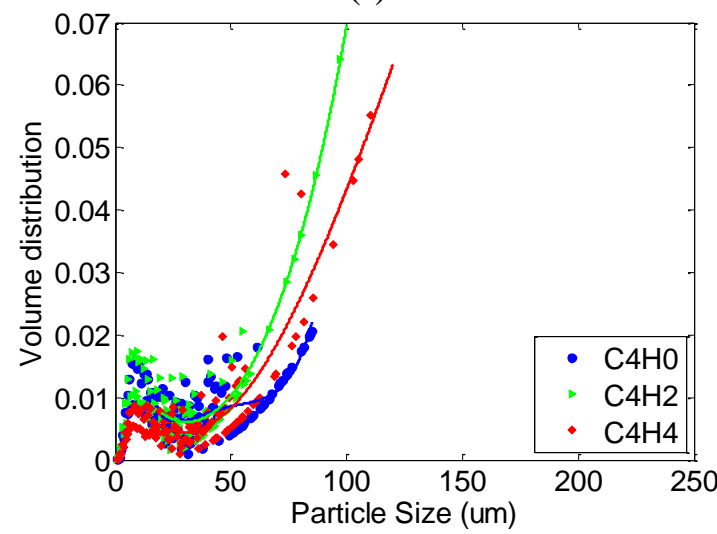

(h)

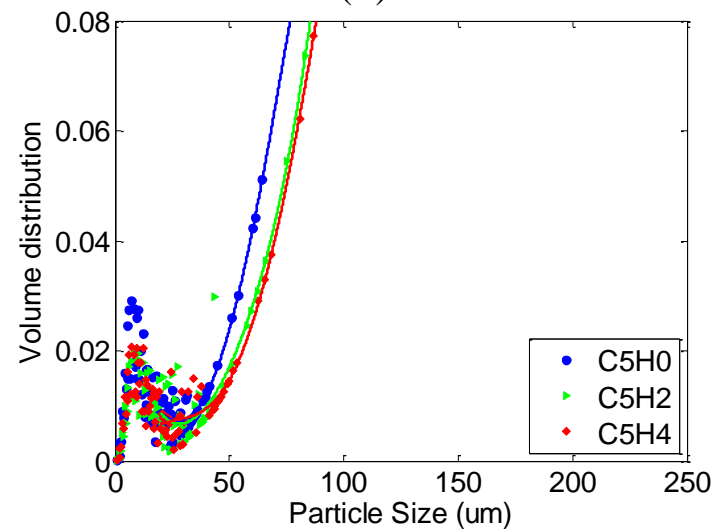

(j)

In this study, it is assumed that strong flocculation is broken at 900 RPM agitation for a sample volume used in the bottom-up setup. The dimensional range of the flocculation is between 50 and $250 \mu \mathrm{m}$, and some samples were also observed to be in a state of permanent coagulation (see Figure 5). They are quantitatively extracted by the deflocculation ratio shown by Figure 7 and the results are summarized in Table 5. The initial measurement denoted by the suffix H0 varies among the samples even though the same mixing protocol and time were applied for all mixes. The mixing energy applied to the samples varies with their rheological properties, as shown in Figure 1, and, hence, the initial degree of flocculation depends on the mix proportion. 
Table 5. Volume fraction of strong flocculation (\%).

\begin{tabular}{cccccc}
\hline Time (h) & Mix C1 & Mix C2 & Mix C3 & Mix C4 & Mix C5 \\
\hline 0 & 16.31 & 12.60 & 21.73 & 16.53 & 10.48 \\
2 & 16.31 & 12.60 & 21.73 & 16.53 & 10.48 \\
4 & 29.15 & 20.78 & 20.67 & 24.97 & 19.41 \\
\hline
\end{tabular}

The $w / \mathrm{cm}$ effect is not trivial: The mix with $w / \mathrm{cm} 30 \%$ (C4) yielded a similar increase of strong flocculation compared to the control mix $\mathrm{C} 1(w / \mathrm{cm} 40 \%)$. In particular, the initial measurements of strong flocculation are approximately identical for mixes $\mathrm{C} 1$ and $\mathrm{C} 4$ even though the viscosity of mix $\mathrm{C} 4$ is around twice that of mix C1 (see Figure 1). Similar to the case of permanent coagulation, a threshold of $w / \mathrm{cm}$ exists for the initial state of strong flocculation (at $\mathrm{H} 0$ ). A mix having a lower $w / \mathrm{cm}$ than the threshold value experiences a similar amount of strong flocculation: The strong flocculation is about $16 \%$ for a mix having a $w / \mathrm{cm}$ less than $40 \%$. For those mixes, their rheology highly depends on their concentration (solid volume fraction) rather than flocculation and the mixing protocol. Increasing $w / \mathrm{cm}$ up to $60 \%$, as in the case of mix C5, significantly reduces the strong flocculation, as expected. Less packing in a mix results in less flocculation. However, the rate of strong flocculation does not follow the effect of $w / \mathrm{cm}$. The increases for $4 \mathrm{~h}$ are 13\%, 8\%, and $9 \%$ for mixes $\mathrm{C} 1, \mathrm{C} 4$, and $\mathrm{C} 5$, respectively. In the superplasticized mixes, $\mathrm{C} 2$ and $\mathrm{C} 3$, the initial amount of strong flocculation and the increase of strong flocculation are not bounded by mixes $\mathrm{C} 1$ and $\mathrm{C} 5$, where their viscosities were bounded by mixes C1 and C5 (see Figure 1). Mix C3, having high-dosage HRWRA, does not show an increase of strong flocculation, consistent with the status of its permanent coagulation. The rheology of those mixes is probably dependent on the particle dispersion rather than on strong flocculation. The particle dispersion would be distributed in the form of weak flocculation.

\subsection{Microstructural Buildup}

In the previous sections, the amounts of permanent coagulation and strong flocculation, respectively, were evaluated. The remaining wet powder in a mix stays in the form of a weakly flocculated network. The weak flocculation is easily broken by external shearing, which results in thixotropy. The values listed in Tables 4 and 5 show the evolution of permanent coagulation and strong and weak flocculation, as is also shown in Figure 9.

Comparing the viscosity with the amount of coagulation makes a link between the microstructure of cement suspension and its mechanical property. As reported in Figure 1, the viscosity of cement suspension decreases with increasing $w / \mathrm{cm}$ ratio and incorporating HRWRA. The amount of permanent coagulation follows the order of viscosity but that of strong flocculation is not in order of viscosity. The rheological protocol to measure the viscosity applies descending rate of shear strain with initial preshearing, which results in break the majority of flocculation. The measured flow curve, therefore, represents the deflocculated state of suspension disregarding its thixotropy. In the deflocculated state, the mechanical property of suspension is determined by the characteristics of permanent coagulation. Such an argument for strong and weak flocculation can be made with the measurement of thixotropic behavior. Picking the thixotropy on the flow curve needs a special rheological protocol, which is beyond the scope of the paper and will be continued in future study. 
Figure 9. Ratio of suspended particles and its evolution according to cement hydration: (a) $\mathrm{C} 1$; (b) $\mathrm{C} 2$; (c) $\mathrm{C} 3$; (d) $\mathrm{C} 4$; and (e) $\mathrm{C} 5$.

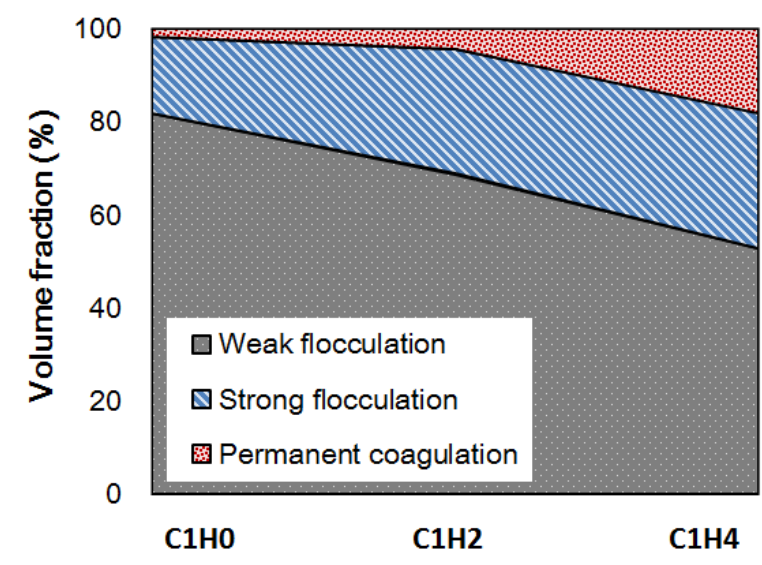

(a)

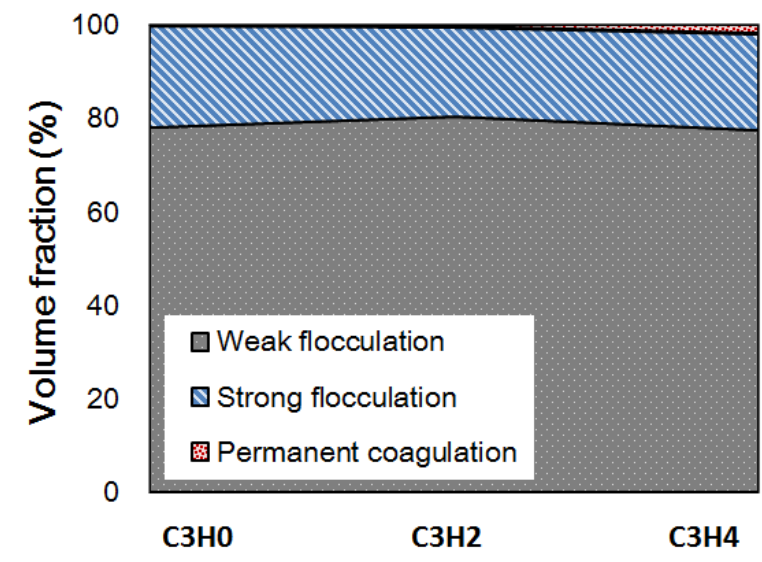

(c)

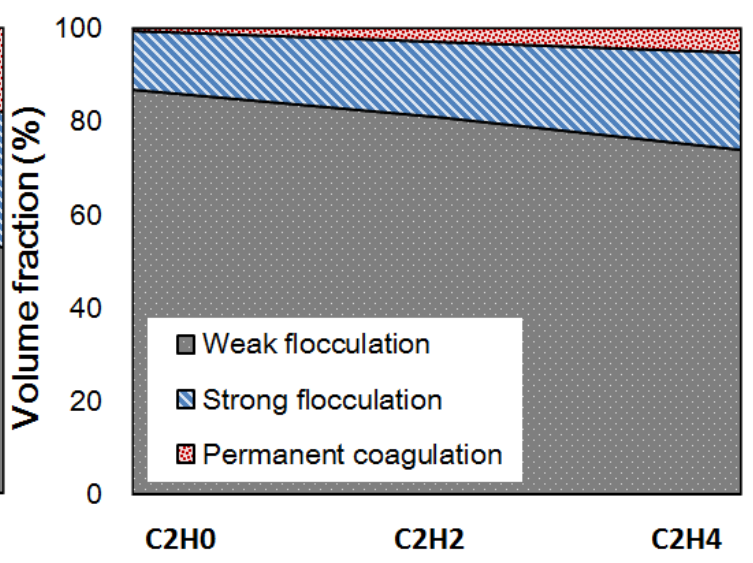

(b)

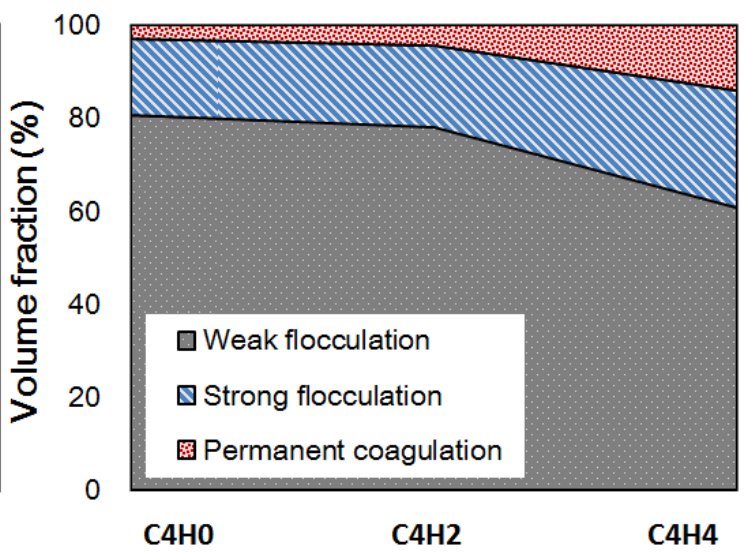

(d)

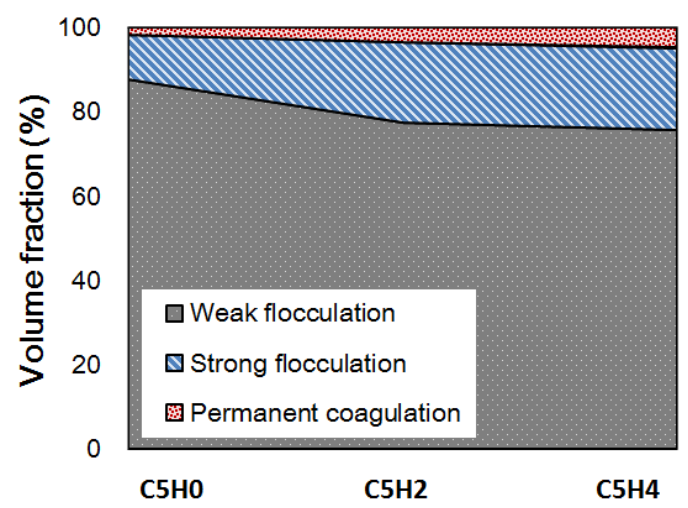

(e)

\section{Conclusions}

This study investigates the mechanical property of suspension of cementitious materials. The measured viscosity is related to coagulation of cement particles and then their coagulation was monitored. Two different setups were proposed to measure the permanent coagulation and strong flocculation separately. The permanent coagulation is responsible for an increase of cement particles up to $12 \mu \mathrm{m}$ while the mean size of the raw powder is $3.8 \mu \mathrm{m}$. The effect of mix proportion on the size is minimal but a certain threshold of $w / \mathrm{cm}$ exists with respect to the rate of permanent coagulation. The 
increase of permanent coagulation is constant for a mix produced with a lower $w / \mathrm{cm}$ that the threshold. In this study, a $w / \mathrm{cm}$ lower than $40 \%$ was considered. The threshold also applies to strong flocculation distributed between 50 and $250 \mu \mathrm{m}$. Initial measurement of strong flocculation is constant for the mixes with lower $w / \mathrm{cm}$ than the threshold. The use of HRWRA diminishes both permanent coagulation and strong flocculation. For a mix having high dosage coagulation was not observed. Finally, from observation of particle sizes in the cement paste, the microstructural buildup was classified into three categories: permanent coagulation and strong and weak flocculation.

\section{Acknowledgments}

This research was supported by Basic Science Research Program through the National Research Foundation of Korea (NRF) funded by the Ministry of Science, ICT \& Future Planning (NRF-2012R1A1A1005402).

\section{Author Contributions}

Hong Jae Yim was involved in the experimental study including data analysis and he prepared the manuscript. Jae Hong Kim designed the project, and contributed on the discussion and conclusion. He also revised the manuscript.

\section{Conflicts of Interest}

The authors declare no conflict of interest.

\section{References}

1. Banfill, P.F.G. The rheology of fresh cement and concrete. Rheol. Rev. 2006, 61-130.

2. Wallevik, J.E. Thixotropic investigation on cement paste: Experimental and numerical approach. J. Non Newton. Fluid Mech. 2005, 132, 86-99.

3. Erdoğan, S.T.; Nie, X.; Stutzman, P.E.; Garboczi, E.J. Micrometer-scale 3-D shape characterization of eight cements: Particle shape and cement chemistry, and the effect of particle shape on laser diffraction particle size measurement. Cem. Concr. Res. 2010, 40, 731-739.

4. Hobbel, E.F.; Davies, R.; Rennie, F.W.; Allen, T.; Butler, L.E.; Waters, E.R.; Smith, J.T.; Sylvester, R.W. Modern Methods of On-Line Size Analysis for Particulate Process Streams. Part. Part. Syst. Charact. 1991, 8, 29-34.

5. Williams, R.A.; Peng, S.J.; Naylor, A. In situ measurement of particle aggregation and breakage kinetics in a concentrated suspension. Powder Technol. 1992, 73, 75-83.

6. Sparks, R.G.; Dobbs, C.L. The use of laser backscatter instrumentation for the on-line measurement of the particle size distribution of emulsions. Part. Part. Syst. Charact. 1993, 10, 279-289.

7. Heath, A.R.; Fawell, P.D.; Bahri, P.A.; Swift, J.D. Estimating average particle size by focused beam reflectance measurement (FBRM). Part. Part. Syst. Charact. 2002, 19, 84-95.

8. Peda, S.; Smieszek, M.; Stollberg, C.; Ay, P. Interpretation of fbrm and 3d orm smf data via simulated nucleation and crystal growth. Laser 2010, 14, 1-55. 
9. Heinrich, J.; Ulrich, J. Application of Laser-Backscattering Instruments for In Situ Monitoring of Crystallization Processes-A Review. Chem. Eng. Technol. 2012, 35, 967-979.

10. Ferron, R.D.; Shah, S.P.; Fuente, E.; Negro, C. Aggregation and breakage kinetics of fresh cement paste. Cem. Concr. Res. 2013, 50, 1-10.

11. Ferron, R.D.; Shah, S.P. The fresh state. Transp. Res. Rec. 2010, 2141, 89-91.

12. Negro, C.; Sánchez, L.M.; Fuente, E.; Blanco, Á.; Tijero, J. Polyacrylamide induced flocculation of a cement suspension. Chem. Eng. Sci. 2006, 61, 2522-2532.

13. Negro, C.; Blanco, A.; Pío, I.S.; Tijero, J. Methodology for flocculant selection in fibre-cement manufacture. Cem. Concr. Compos. 2006, 28, 90-96.

14. Jarabo, R.; Fuente, E.; Moral, A.; Blanco, Á.; Izquierdo, L.; Negro, C. Effect of sepiolite on the flocculation of suspensions of fibre-reinforced cement. Cem. Concr. Res. 2010, 40, 1524-1530.

15. American Society for Testing and Materials (ASTM). Standard Guide for Measurement of the Rheological Properties of Hydraulic Cementious Paste Using a Rotational Rheometer, 2012; Annual Book of ASTM Standards, ASTM C1749-12; ASTM: West Conshohocken, PA, USA, 2012.

16. Thomas, D.N.; Judd, S.J.; Fawcett, N. Flocculation modelling: A review. Water Res. 1999, 33, 1579-1592.

(C) 2014 by the authors; licensee MDPI, Basel, Switzerland. This article is an open access article distributed under the terms and conditions of the Creative Commons Attribution license (http://creativecommons.org/licenses/by/3.0/). 